\title{
Tendencias en las tasas de incidencia de cáncer colorrectal en Navarra en el periodo 1990-2005
}

\author{
Incidence rate trends for colorectal cancer in Navarre \\ (North of Spain) in the 1990-2005 period
}

\author{
J. Etxeberria ${ }^{1,3}$, M.D. Ugarte ${ }^{1}$, A. Barricarte ${ }^{2,3}$, T. Goicoa ${ }^{1}$, C. Moreno-Iribas ${ }^{2,3}$, \\ M. J. Azagra ${ }^{2}$, E. San Román ${ }^{2}$, R. Burgui ${ }^{2,3}$, A.F. Militino ${ }^{1}$, E. Ardanaz ${ }^{2,3}$
}

\section{RESUMEN}

Fundamento. En España, se ha observado un aumento de la incidencia de cáncer colorrectal (CCR) en ambos sexos en los últimos años, posiblemente debido a las mejoras diagnósticas, a la occidentalización de la dieta y al empeoramiento de los niveles de obesidad entre otros. En este trabajo se han estudiado las tendencias de la incidencia de CCR en las diferentes áreas de salud de Navarra (norte de España) durante el período 1990-2005.

Métodos. Para cada sexo y área, se obtuvieron las tendencias de las tasas de incidencia y los correspondientes intervalos de confianza mediante modelos de P-splines.

Resultados. Se observa una tendencia creciente de la incidencia de CCR en la mayoría de las áreas para ambos sexos, siendo menos pronunciada en las mujeres que en los hombres. En la zona centro de Pamplona (la capital) se observa una tendencia decreciente para los hombres durante el período estudiado.

Conclusiones. Para cambiar las tendencias crecientes observadas en la mayoría de las áreas de la provincia, la prevención primaria es la mejor estrategia. Sin embargo, adquirir estilos de vida saludables tiene resultados a largo plazo por lo que un programa de detección temprana serviría como estrategia de prevención a más corto plazo.

Palabras clave. Cáncer colorrectal. Incidencia. Tendencias. Modelo P-spline.

\begin{abstract}
Background. In Spain, an increase in the incidence of colorectal cancer (CRC) has been observed in both sexes in recent years, probably due to an improved diagnostic, the westernization of dietary habits, and worse obesity levels, among others factors. In this work, CRC incidence rate trends in different health areas in Navarre (northern Spain) are studied during the 1990-2005 period.
\end{abstract}

Methods. An estimated incidence trend curve for each health area and the corresponding confidence bands were obtained for each gender using P-spline models.

Results. These results show an increasing trend of CRC in most of the areas in both sexes, being less pronounced in women than in men. In the central area of Pamplona (the capital city) a decreasing trend has been observed for men during the studied period.

Conclusions. Primary prevention is the best strategy to change the increasing trend observed in most areas of the province of Navarre. However, a healthy lifestyle has long-term results, so it is important to have an early detection program that would serve as a short-term prevention strategy.

Key words. Colorectal cancer. Incidence. Temporal trends. P-spline models.
1. Departamento de Estadística de I.O., Universidad Pública de Navarra.

2. Instituto de Salud Pública de Navarra.

3. Consorcio de Investigación Biomédica en Red especializado en Epidemiología y Salud Pública. (CIBERESP).

Recepción: 23 de septiembre de 2011 Aceptación provisional: 2 de noviembre de 2011 Aceptación definitiva: 6 de febrero de 2012

\section{Correspondencia:}

Departamento de Estadística de I.O.

Universidad Pública de Navarra

Campus de Arrosadía

31006 Pamplona (Navarra)

E-mail: jaione.etxeberria@unavarra.es

Financiación: Este trabajo ha sido financiado por el Ministerio de Ciencia e Innovación (Proyecto MTM200803085 y MTM2011-22664) y por el CIBER de Epidemiología y Salud Pública (CIBERESP). 


\section{INTRODUCCIÓN}

Según datos publicados por la Agencia Internacional para la Investigación sobre el Cáncer (IARC) en 2008, el cáncer colorrectal (CCR) es el cáncer más frecuente en cifras absolutas en el mundo. En hombres es el cuarto más frecuente y el tercero en las mujeres ${ }^{1-3}$. Dado que el cáncer colorrectal es más frecuente en Australia, Nueva Zelanda, Europa y otros países desarrollados, se considera una enfermedad con factores de riesgo asociados a un estilo de vida occidental. En las últimas décadas la incidencia de CCR mostró una tendencia decreciente en algunos países desarrollados como el Reino Unido, Francia, EE.UU. y Australia, que contrastan con lo que ocurrió en los países en transición económica en el mundo (entre ellos Espa$\tilde{n} a^{4}$ ), donde se observó un notable incremento de las tasas ${ }^{5,6}$.

Los tumores malignos de colon y recto se sitúan en España como el segundo cáncer con mayor incidencia entre las mujeres después del cáncer de mama (excluyendo el cáncer de piel no melanoma) y el segundo en los hombres después del cáncer de pulmón o el tercero después de pulmón y de próstata ${ }^{7}$ dependiendo del área. Además, su incidencia parece ir en aumento en los últimos años. Las tasas publicadas para el período 1998-2002 por los diferentes registros de cáncer españoles ${ }^{7}$ evidenciaron diferencias por áreas geográficas y por género. La edad se convierte en un factor de riesgo después de los 50 años, siendo a partir de aquí el riesgo de 1,5 a 2 veces mayor cada década. El 92,5\% de los casos ocurren por encima de 50 años de edad y el $78 \%$ se diagnostican antes de los 80 años ${ }^{8}$.

Las tasas de incidencia de cáncer colorrectal observadas entre los hombres de Navarra se encuentran en torno a la media europea mientras que las tasas de las mujeres son considerablemente más bajas que las observadas en los países industrializados de Europa o Estados Unidos ${ }^{9}$.

Las distintas poblaciones a nivel mundial experimentan diferentes frecuencias de cáncer colorrectal y éstas cambian con el tiempo. Además, poblaciones con estilos de vida distintos en una misma comunidad presentan frecuencias distintas. Se sabe también que los grupos de migrantes pierden rápidamente el riesgo asociado a su comunidad de origen y adquieren el patrón de la nueva comunidad. Por estas razones, se cree que el cáncer colorrectal es una enfermedad ambiental, definida por las prácticas culturales y sociales y por los estilos de vida. Hasta un 70-80\% de los cánceres colorrectales deben su aparición a estos factores, por lo que este cáncer es una de las principales neoplasias en las que las causas pueden ser rápidamente identificadas, y una gran parte de la enfermedad es teóricamente evitable ${ }^{10}$. En este contexto, resulta de interés investigar las tendencias y las diferencias geográficas del CCR de Navarra. El objetivo del estudio es analizar las tendencias de las tasas de incidencia de cáncer colorrectal en las distintas áreas de Navarra en el periodo 1990-2005.

\section{MÉTODOS}

Se consideraron los casos incidentes de cánceres de colon y recto registrados por el Registro del Cáncer de Navarra ${ }^{11}$ que se corresponden con los códigos C18C21 de la Clasificación Internacional de Enfermedades $10^{\mathrm{a}}$ edición (CIE-10) ${ }^{12}$. Para la evaluación de los criterios de calidad del registro se consideraron los siguientes criterios: el porcentaje de casos identificados por certificados de defunción (CDOs), el porcentaje de casos identificados por investigación clínica y el de confirmados por verificación histológica ${ }^{9}$. En la tabla 1 se muestran los criterios de calidad, casos sin edad y la tasa de letalidad (razón de mortalidad/incidencia) del registro de cáncer de Navarra.

En los datos utilizados para el estudio de tendencias se incluyeron el número de tumores diagnosticados entre 1990-2005, por año y grupo de edad (0-4, 5-9, ..., 80-84, $85+$ ) al diagnóstico, divididos por hombres y mujeres en cada una de las 54 zonas básicas de salud de Navarra según lo establecido en la Ley Foral 22/1985 de zonificación sanitaria $^{13}$ y en las ampliaciones de la misma. Los datos sobre la población fueron 
obtenidos del Instituto de Estadística de Navarra. Para llevar a cabo el estudio de tendencias, se realizaron agrupaciones de las zonas básicas de salud. El número de áreas se redujo de 54 a 7 . Se consideraron cuatro grandes áreas fuera de la capital, según la distribución geográfica de Navarra y tres áreas dentro de Pamplona. Las áreas definidas fueron las siguientes: zona norte (área pirenaica), zona oriental, zona occidental, zona sur (la región del Ebro) y tres áreas más dentro de Pamplona: zona Norte compuesta por San Jorge, Rochapea, Ansoain y Chantrea, zona central agregando Casco Viejo, Ensanche I y II y finalmente la zona sur de Pamplona que agrupa a La Milagrosa, Azpilagaña, Mendillorri, Iturrama, San Juan y Ermitagaña. Dado que gran parte de la población de Navarra se concentra en la capital (alrededor del 31\%) el mayor número de casos pertenecen a las áreas de salud de Pamplona. Por ello, se decidió desagregar Pamplona y agrupar las zonas básicas de salud del resto de Navarra.

Tabla 1. Indicadores de calidad del cáncer colorrectal por sexo y año de diagnóstico para los periodos $1990-1997$ y $1998-2005$

\begin{tabular}{|c|c|c|c|c|c|c|c|c|}
\hline & \multicolumn{4}{|c|}{ Hombres } & \multicolumn{4}{|c|}{ Mujeres } \\
\hline & \multicolumn{2}{|c|}{ 1990-1997 } & \multicolumn{2}{|c|}{ 1998-2005 } & \multicolumn{2}{|c|}{ 1990-1997 } & \multicolumn{2}{|c|}{ 1998-2005 } \\
\hline & Casos & $\%$ & Casos & $\%$ & Casos & $\%$ & Casos & $\%$ \\
\hline $\mathrm{CDOs}^{*}$ & 45 & 3,48 & 20 & 1,14 & 61 & 6,57 & 22 & 1,82 \\
\hline Clínico e Investigación Clínica & 44 & 3,40 & 58 & 3,30 & 50 & 5,38 & 82 & 6,78 \\
\hline Verificación Histológica & 1204 & 93,12 & 1682 & 95,57 & 818 & 88,05 & 1106 & 91,40 \\
\hline Total & 1293 & 100 & 1760 & 100 & 929 & 100 & 1210 & 100 \\
\hline Razón Mortalidad/Incidencia en \% & & 48,41 & & 41,59 & & 50,70 & & 48,93 \\
\hline Fecha de Nacimiento conocida en\% & & 100 & & 100 & & 100 & & 100 \\
\hline
\end{tabular}

${ }^{*}$ Casos conocidos solo por certificados de defunción

Se calcularon tasas de incidencia de cáncer colorrectal ajustadas por edad utilizando población mundial (TAE(M)) para hombres y para mujeres por separado. Para estudiar las tendencias temporales de las tasas estandarizadas por edad se utilizó un modelo de P-splines. Un modelo de Psplines permite modelizar la tasas ajusta- das del área i como una función suave f del tiempo $t$ donde toma valores en el intervalo 1990-2005. La función f se estima utilizando P-splines con bases de B-splines ${ }^{14}$. Concretamente, las tasas ajustadas por edad en el área i y en el año t se modelizan de la manera siguiente:

$$
\mathrm{TAE}_{\mathrm{it}}=\mathrm{f}_{\mathrm{i}}(\mathrm{t})+\varepsilon_{\mathrm{it}} ; \varepsilon_{\mathrm{it}} \sim \mathrm{N}\left(0, \sigma_{\varepsilon}\right) \text { donde } \mathrm{t}=1990, \ldots, 2005, \mathrm{i}=1, \ldots, 7
$$

En este trabajo se calcula además el porcentaje de cambio anual (PCA) de las tasas de incidencia a lo largo del perio- do 1990-2005 para cada área y su correspondiente intervalo de confianza al 95\% (IC95\%( PCAi) ) siguiendo la expresión:

$$
\mathrm{PCA}_{\mathrm{i}}=\left(\exp \left(\mathrm{b}_{1}\right)-1\right) * 100 ; \mathrm{IC}_{95 \%}\left(\mathrm{PCA}_{\mathrm{i}}\right)=\left[\exp \left(\mathrm{IC}_{95 \%}\left(\mathrm{~b}_{1}\right)\right)-1\right]^{*} 100
$$


donde $b_{1}$ es la pendiente de la siguiente recta ajustada en cada área utilizando como variable respuesta el logaritmo de las tasas suavizadas obtenidas del modelo (1). Esto es,

$$
\log \left(\mathrm{TAE}_{\mathrm{it}}\right)=\mathrm{b}_{0}+\mathrm{b}_{1} \mathrm{t}+\varepsilon_{\mathrm{it}} ; \varepsilon_{\mathrm{it}} \sim \mathrm{N}\left(0, \sigma_{\varepsilon}\right) \text { donde } \mathrm{t}=1990, \ldots, 2005
$$

El análisis y los gráficos se realizaron utilizando el software estadístico R 2.14.1 ${ }^{15}$.

\section{RESULTADOS}

Se registraron un total de 5.192 casos de cáncer colorrectal en la base de datos entre 1990 y 2005 , de los cuales 3.053 $(58,8 \%)$ fueron hombres y $2.139(41,2 \%)$ mujeres. En la tabla 2 se muestra el número total de casos observados en el periodo 1990-2005 y la población a mitad de periodo de cada una de las áreas divididas por grupos de edad (<50, 50-64,>64) y género. La tabla 3 muestra el porcentaje de cambio anual (PCA) de las tasas ajustadas por edad (suavizadas) por sexo y área con el correspondiente intervalo de confianza. En hombres se observa un aumento de las tasas de CCR en la mayoría de las áreas que varían entre el $1,21 \%$ y $4,54 \%$ dependiendo del área. Como excepción, la zona centro de Pamplona donde las tasas disminuyeron un $1,37 \%(1,343 \%-1,387 \%)$ anual. Entre las mujeres, las tasas se incrementaron entre un $0,7 \%$ y $4,9 \%$ anual en la mayoría de las zonas exceptuando el área sur de $\mathrm{Na}$ varra, en la que se observó un decremento del $0,54 \%(0,539 \%-0,546 \%)$. En la figura 1 se muestra la representación gráfica de las tendencias de las tasas específicas por grupos de edad y género por cada 100.000 habitantes a los largo del periodo de estudio. Entre todos los grupos estudiados, los hombres > 64 son los que mayores tasas específicas presentan seguidos por las mujeres $>64$, los hombres de entre 50-64 y las mujeres de este mismo grupo de edad. Los hombres y mujeres menores de 50 son los que menores tasas presentan a lo largo del periodo de estudio.

Tabla 2. Total de casos acumulados en el periodo 1990-2005 y población por grupos de edad, género y área a mitad de periodo

\begin{tabular}{|c|c|c|c|c|c|c|c|c|c|c|c|c|}
\hline \multirow[b]{3}{*}{ Zona } & \multicolumn{6}{|c|}{ HOMBRES } & \multicolumn{6}{|c|}{ MUJERES } \\
\hline & \multicolumn{2}{|c|}{ < 50 Años } & \multicolumn{2}{|c|}{ 50-64 Años } & \multicolumn{2}{|c|}{ > 64 Años } & \multicolumn{2}{|c|}{$<50$ Años } & \multicolumn{2}{|c|}{ 50-64 Años } & \multicolumn{2}{|c|}{ > 64 Años } \\
\hline & Casos & Pobl. & Casos & Pobl. & Casos & Pobl. & Casos & Pobl. & Casos & Pobl. & Casos & Pobl. \\
\hline Norte & 43 & 38641 & 208 & 10949 & 252 & 4627 & 24 & 34677 & 102 & 9721 & 199 & 6346 \\
\hline Este & 26 & 34952 & 227 & 10650 & 354 & 5411 & 17 & 32653 & 120 & 10367 & 268 & 7577 \\
\hline Oeste & 17 & 19186 & 124 & 6883 & 220 & 3984 & 11 & 17415 & 70 & 6680 & 148 & 5611 \\
\hline Sur & 31 & 26589 & 150 & 8654 & 258 & 4614 & 19 & 25013 & 101 & 8840 & 214 & 6618 \\
\hline Pamplona Norte* & 21 & 17838 & 125 & 5994 & 157 & 2168 & 9 & 17094 & 77 & 6147 & 110 & 3154 \\
\hline Pamplona Centro* * & 9 & 9206 & 86 & 3100 & 156 & 1962 & 11 & 9174 & 62 & 4267 & 175 & 4134 \\
\hline Pamplona Sur* * * & 48 & 32904 & 247 & 10757 & 294 & 4054 & 31 & 33587 & 142 & 12114 & 229 & 6356 \\
\hline
\end{tabular}

\footnotetext{
*San Jorge, Rochapea, Ansoain y Chantrea

**Casco Viejo, Ensanche I y II

***La Milagrosa, Azpilagaña, Mendillorri, Iturrama, San Juan y Ermitagaña
} 
Tabla 3. Porcentaje de cambio anual (PCA) en el periodo 1990-2005 de las tasas ajustadas (suavizadas) y el correspondiente intervalo de confianza (IC) al 95\% de nivel de confianza para las diferentes zonas

\begin{tabular}{|c|c|c|c|c|c|c|}
\hline \multirow[b]{3}{*}{ Zona } & \multicolumn{3}{|c|}{ Hombres } & \multicolumn{3}{|c|}{ MUJERES } \\
\hline & \multicolumn{3}{|c|}{$\begin{array}{l}\text { Porcentaje de cambio anual (PCA) } \\
\text { 1990-2005 }\end{array}$} & \multicolumn{3}{|c|}{$\begin{array}{c}\text { Porcentaje anual de cambio (PCA) } \\
1990-2005\end{array}$} \\
\hline & \multirow{2}{*}{$\frac{\text { PCA }}{4,54}$} & \multicolumn{2}{|c|}{ IC del PCA 95\% } & \multirow{2}{*}{$\begin{array}{r}\text { PCA } \\
1,82\end{array}$} & \multicolumn{2}{|c|}{ IC del PCA 95\% } \\
\hline Norte & & 4,292 & 4,781 & & 1,778 & 1,855 \\
\hline Este & 1,44 & 1,415 & 1,463 & 0,71 & 0,701 & 0,713 \\
\hline Oeste & 4,19 & 3,980 & 4,395 & 4,90 & 4,613 & 5,184 \\
\hline Sur & 1,69 & 1,658 & 1,726 & $-0,54$ & $-0,546$ & $-0,539$ \\
\hline Pamplona Norte* & 2,36 & 2,299 & 2,431 & 1,61 & 1,578 & 1,639 \\
\hline Pamplona Centro** & $-1,37$ & $-1,387$ & $-1,343$ & 1,52 & 1,495 & 1,550 \\
\hline Pamplona Sur* * * & 1,21 & 1,192 & 1,226 & 1,65 & 1,614 & 1,678 \\
\hline Total Navarra & 2,24 & 0,987 & $\mathbf{3 , 5 0 7}$ & 1,55 & 0,278 & 2,828 \\
\hline
\end{tabular}

*San Jorge, Rochapea, Ansoain y Chantrea

**Casco Viejo, Ensanche I y II

***La Milagrosa, Azpilagaña, Mendillorri, Iturrama, San Juan y Ermitagaña

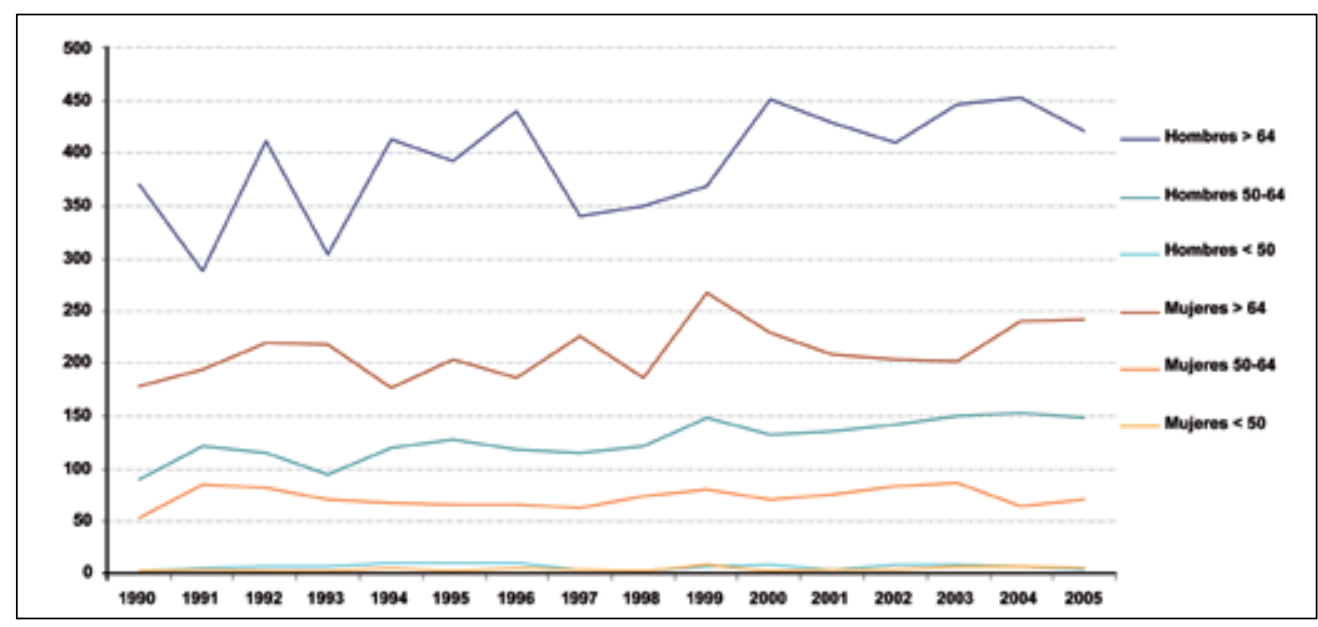

Figura 1. Tendencias de las tasas de incidencia específicas por edad y sexo en Navarra entre 1990 y 2005 de cáncer colorrectal.

Las figuras 2 y 3 muestran las tendencias de las tasas de CCR ajustadas por edad a la población mundial por 100.000 habitantes durante 1990-2005 para hombres y mujeres respectivamente. En cada figura, además de las tasas de incidencia y la tendencia temporal, se han añadido las bandas de confianza al 95\% para la tendencia en todo el periodo 1990-2005. Los puntos negros se corresponden con las tasas estandarizadas por edad. Las líneas azules representan las tasas suavizadas y las bandas grises los intervalos de confianza (IC) al 95\%. También se han añadido en color rojo, la tasa estandarizada por edad para toda Navarra. 


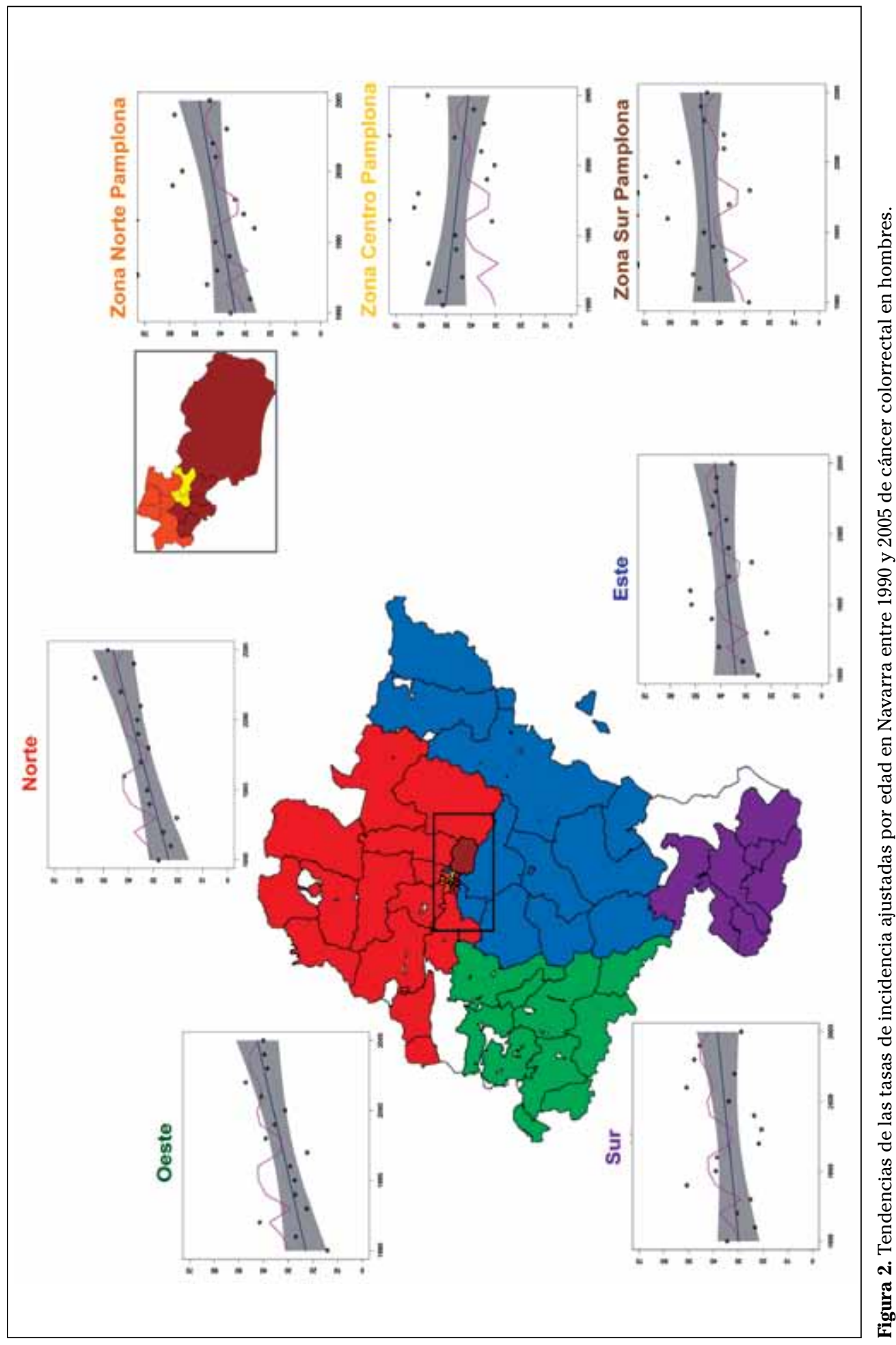




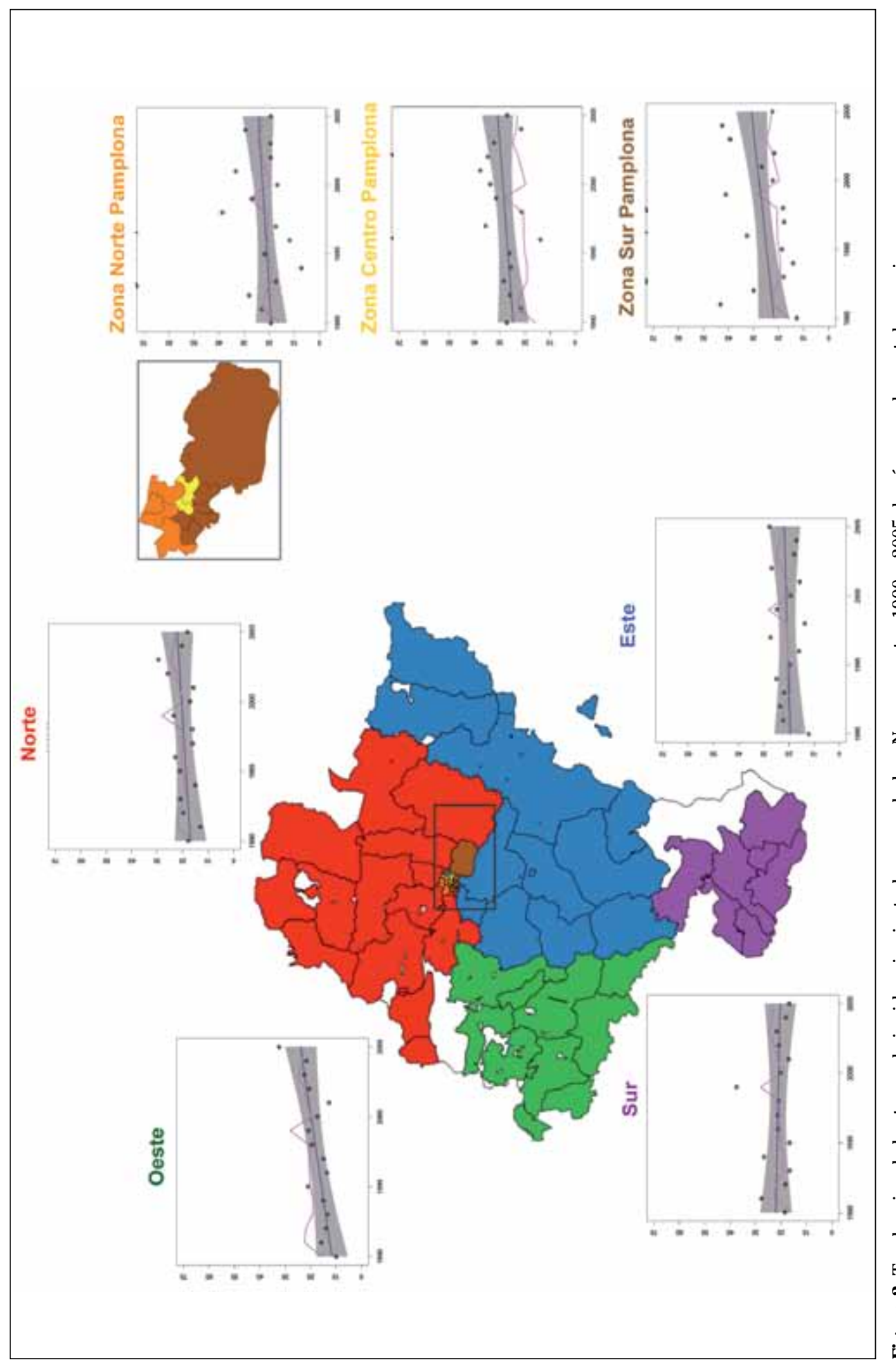

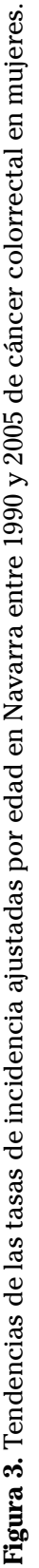


En hombres (Fig. 2), se observa un aumento en las tasas de incidencia en todas las áreas excepto en el centro de Pamplona, donde es apreciable una tendencia descendente del cáncer colorrectal. Es destacable también lo observado en la zona que agrupa los barrios del sur de la capital. Aunque el incremento de las tasas no es tan pronunciado como en otras zonas, las tasas de esta zona sur de Pamplona se sitúan por encima de la tasa de Navarra en todo el periodo. También las tasas de la zona centro de Pamplona se sitúan por encima de la tasa de Navarra hasta finales de periodo. En mujeres (Fig. 3), las representaciones gráficas también muestran una tendencia de las tasas estable o al alza dependiendo de la zona, pero notablemente menos pronunciada que en los hombres. En este caso, la zona que agrupa los barrios del sur y centro de Pamplona se sitúa por encima de la tasa Navarra en mujeres.

\section{DISCUSIÓN}

El análisis de tendencias de las tasas estandarizadas de cáncer colorrectal en cada área muestra por un lado una mayor incidencia en el núcleo urbano de Pamplona en ambos sexos y por otro una tendencia creciente de la incidencia en la mayoría de las áreas, siendo en las mujeres menos pronunciada que en los hombres. Como excepción, en la zona centro de Pamplona (para los hombres) y en la zona sur de Navarra (para las mujeres) se ha producido un descenso del $1,37 \%$ y $0,54 \%$ anual respectivamente. La zona centro de Pamplona, caracterizada por ser una de las zonas económicamente más privilegiadas de $\mathrm{Na}$ varra, es la que presenta tasas más altas en el primer quinquenio y es al mismo tiempo la zona donde la tendencia muestra un patrón diferente. Una posible explicación para esta discrepancia es que esta zona esté presentando una evolución más favorable en la disminución del tabaquismo, obesidad y aumento del ejercicio físico.

Recientemente se ha publicado un estudio en el cual se analizan los patrones espacio-temporales de los riesgos de incidencia de cáncer colorrectal en Navarra ${ }^{16}$.
Es importante notar que en este estudio la zonificación empleada es distinta, se analizan riesgos en lugar de tasas y se utiliza un método de estandarización indirecto, tomando como referencia la población navarra, por lo que los resultados obtenidos no son directamente comparables. En cualquier caso las conclusiones principales de ambos estudios son similares ya que en dicho estudio se observa un aumento de la incidencia del cáncer colorrectal en la mayoría de las áreas geográficas y en ambos géneros, aunque algo menor en mujeres que en hombres.

Existen en la literatura otros estudios diseñados para el estudio de la incidencia de CCR en áreas pequeñas, principalmente en EEUU y proporcionan resultados diversos ${ }^{17}$. Diferencias geográficas en la incidencia pueden reflejar variaciones en los factores de exposición que participan en el proceso de desarrollo del cáncer colorrectal como el consumo excesivo de carne roja y procesada, el consumo excesivo de alcohol, la deficiencia de vitaminas B y D, la obesidad, la inactividad física, la diabetes mellitus, el tabaquismo, los antecedentes familiares de cáncer colorrectal, entre otros o la recepción del tratamiento ${ }^{18}$. En la literatura, los factores asociados al CCR se resumen en cuatro grupos: factores biológicos (hereditarios, patología intestinal y otros); factores medio-ambientales o sociales (de riesgo o de protección); intervenciones médicas y factores etio-patogénicos ${ }^{19}$. El CCR hereditario, afecta solamente a parientes en primer grado (hijo o hermano) ( $R R=$ $1,7)$ y acumula el $20 \%$ de los casos $\mathrm{CCR}^{20}$, lo que puede justificar el seguimiento sistemático preventivo en estas personas. Los demás parientes tienen la misma probabilidad individual de padecer pólipos adenomatosos, adenomas y cáncer que la población en general. Por otro lado, los enfermos que han sido resecados padecen un segundo cáncer colorrectal primario entre el 1,5 y $3 \%^{21}$ en los 5 primeros años del post-operatorio. Del mismo modo los pólipos adenomatosos presentan un RR entre 3,5 y 6,5 de desarrollar $\mathrm{CCR}^{22}$. La existencia de diferencias geográficas en 
los factores de riesgo es una cuestión que debería abordarse en futuros estudios.

Estudios realizados en otros lugares del mundo en los últimos años muestran diferentes situaciones de la incidencia del cáncer colorrectal. En Australia y Europa occidental se ha observado por ejemplo una estabilización de la incidencia de CCR en hombres. En Estados Unidos la incidencia ha disminuido ${ }^{5,6}$. Sin embargo, en Austria, Croacia, Eslovenia, Italia y la República Checa se observa un ligero aumento en la incidencia ${ }^{21,23}$. En mujeres, en Escocia, Irlanda del Norte, o Polonia se observa una estabilización o disminución de la incidencia de $\mathrm{CCR}^{23}$. En España, desde mediados de la década de 1970 se observó un aumento en la incidencia de CCR en ambos sexos ${ }^{6}$, pero más acentuada en los hombres ${ }^{20}$. Esto podría atribuirse a cambios en los hábitos alimenticios de la población española tales como un mayor consumo de azúcar, carne roja y procesada, un menor consumo de fibra $^{24}, \mathrm{y}$ una menor actividad física ${ }^{25}$.

A diferencia de la incidencia, algunos estudios han observado que las tasas de mortalidad de CCR disminuyen en toda Europa y también en España ${ }^{20,21,23}$. La divergencia entre la incidencia y la mortalidad puede ser explicada por una mejora en la supervivencia. La supervivencia del CCR en España se sitúa en el 53,6\% a los 5 años, y 70,7\% a los 5 años en los casos en que los pacientes sobreviven el primer año. La supervivencia tiende a ser ligeramente mayor entre las mujeres que entre los hombres, es decir, $54,5 \%$ versus $53,4 \%$, respectivamente ${ }^{26}$. En Navarra (datos de informe interno), la supervivencia a los 5 años de los pacientes con CCR pasó de 49,2 a $54,7 \%$ en los hombres entre los periodos 1985-1989 y 1995-1999 y de 53,7 a $57,7 \%$ entre las mujeres. Esta mejora en la supervivencia se observa especialmente en pacientes jóvenes, probablemente debido a cambios positivos en el tratamiento de $\mathrm{CCR}^{27}$ o en el diagnóstico. Resultados preliminares de estudios de alta resolución del CCR en los que Navarra está participando indican buenas prácticas clínicas en quimioterapia y administración de la radioterapia neoadyuvante ${ }^{28}$.
Debido al impacto de este tipo de cáncer en España, y especialmente en Navarra, la aplicación de la prevención del CCR y los programas de detección temprana puede ayudar a cambiar esta tendencia de la incidencia y/o mortalidad en las diferentes provincias, tal y como sucedió con el cáncer de mama $^{29}$. Siguiendo las recomendaciones de la Unión Europea ${ }^{30}$ y la estrategia contra el cáncer seguida por el Sistema Nacional de Salud en España, algunas comunidades autónomas españolas como Cataluña, Valencia y Murcia han implantado el programa de detección precoz del $\mathrm{CCR}^{21}$. El rango de edad de la población objetivo es 50-69 años y cada dos años se les realiza un test para la detección de sangre oculta en heces ${ }^{31}$. Navarra tiene previsto iniciar programas de este tipo en el futuro ${ }^{21}$. En este sentido, es de gran interés disponer de información sobre la incidencia de CCR antes y después de la aplicación de los programas de cribado en Navarra. Este estudio podría servir como un ejemplo de las tendencias de las tasas de incidencia de CCR previo a la aplicación de programas preventivos secundarios. Se estima que para 2015 todos los ciudadanos españoles estarán cubiertos por un programa de cribado $^{31}$.

Los registros de incidencia son un instrumento imprescindible para la investigación y control del cáncer. No sólo permiten conocer la incidencia y su evolución, sino que constituyen, junto a los registros de mortalidad, un elemento clave en la vigilancia epidemiológica del cáncer y también del ambiente ${ }^{32}$. Por lo tanto, es importante mantener y potenciar los registros poblaciones de cáncer ${ }^{33}$.

En resumen, se observa un aumento en la incidencia de CCR en la mayoría de las áreas de Navarra tanto en hombres como en mujeres. El análisis del CCR en áreas pequeñas de Navarra permitirá a los investigadores en salud y los responsables locales conocer la situación de la incidencia de este cáncer en las distintas regiones que componen la Comunidad Foral de Navarra. Se plantea un desafío considerable cuando se trata de prevención primaria, de aplicación de los programas de cribado de la población, y de planificación de la atención 
oncológica en los próximos años en España $a^{21}$ y en sus comunidades autónomas. Mientras tanto, es necesario continuar promocionando estilos de vida saludables para la prevención primaria en hombres y mujeres de todas las edades.

\section{Agradecimientos}

Queremos expresar nuestro más sincero agradecimiento a todos los servicios y profesionales del Sistema Sanitario de Navarra, tanto de centros públicos como privados, que de una u otra manera han colaborado y colaboran con el Registro de Cáncer de Navarra. Nuestro reconocimiento por su inestimable trabajo en la recopilación, la codificación y la revisión de los datos a lo largo de los últimos años a $\mathrm{M}^{\mathrm{a}}$ Eugenia Pérez de Rada, Carmen Ezponda, Nieves Navaridas (†) y Remedios Pérez. Y así mismo agradecer a los miembros de la Comisión Asesora Técnica del Registro de Cáncer de Navarra su participación y colaboración con el Registro.

\section{BIBLIOGRAFÍA}

1. PARKIN DM. International variation. Oncogene 2004; 23: 6329-6340.

2. Parkin DM, Bray F, Ferlay J, Pisani P. Global cancer statistics, 2002. CA Cancer J Clin 2005; 55 : 74-108.

3. Ferlay J, Shin HR, Bray F, Forman D, Mathers C, PARKIN DM. GLOBOCAN 2008, Cancer Incidence and Mortality Worldwide: IARC CancerBase No. 10. Lyon, France: International Agency for Research on Cancer; 2010.

4. Béjar Prado LM, Gili M, Ramírez G, López J, CaBANILLAS JL. Dietary changes and colorrectal cancer trends in Spain during 1951-2007. Rev Esp Enferm Dig 2010; 102: 159-168.

5. Edwards, BK, Ward E, Kohler BA, Eheman C, ZAUBER AG, ANDERson RN et al. Annual report to the nation on the status of cancer, 19752006, featuring colorrectal cancer trends and impact of interventions (risk factors, screening, and treatment) to reduce future rates. Cancer 2010; 116: 544-573.

6. Center MM, Jemal A, WARD E. International trends in colorrectal cancer incidence rates. Cancer Epidemiol. Biomarkers Prev 2009; 18 : 1688-1694.
7. Curado MP, Edwards B, Shin HR, Storm H, FerLAY J, HEAnue $M$ et al. Cancer incidence in five continents. Vol. IX. Lyon: IARC Press; 2007.

8. Registro de CÁNCER de NAVARRA. Incidencia y mortalidad por cáncer en Navarra, 19931997. Tendencias en los últimos 25 años. An Sist Sanit Navar 2001; 24: 339-362.

9. Ardanaz E, Moreno-Iribas C, Pérez de Rada ME, EzPonda C, Floristán Y, NAVARIDAs N et al. Incidencia y mortalidad por cáncer en Navarra, 1998-2002: Evolución en los últimos 30 años. An Sist Sanit Navar 2007 30: 245-270.

10. Boyle P, LANGMAN JS. ABC of colorectal cancer: Epidemiology. BMJ 2000; 321: 805-808.

11. Ardanaz E, Barricarte A, Pérez de Rada ME. EzPonda C, Navaridas N. Cancer incidence in Navarra 1998-2002. En: Curado MP, Edwards B, Shin HR, Storm H, Ferlay J, Heanue M et al., editores. Cancer incidence in five continents. Vol. IX. Lyon: IARC Press; 2007.

12. International Organization Health. ICD-10a. International Statistical Classification of Diseases and related Health problems.

13. LEY FORAL 22/1985, de 13 de noviembre, por la que se establece la zonificación sanitaria de Navarra. (BON 110 de 20 de noviembre de 1985).

14. EiLERS PHC, Marx BD. Flexible smothing with B-splines and penalties. Statistical Science 1996; 11: 89-121.

15. R Development Core Team (2011). R: A language and environment for statistical computing. R Foundation for Statistical Computing, Vienna, Austria. http://www.r-project. org/. (fecha consulta 22/12/2011)

16. Ugarte MD, Etxeberria J, Goicoa T, Ardanaz E. Gender-specific spatio-temporal patterns of colorectal cancer incidence in Navarre, Spain (1990-2005). Cancer Epidemiology. 2012; doi: 10.1016/j.canep.2011.10.004.

17. Fournel I, Cottet V, Binquet C, Jooste V, Faivre J, Bouvier AM et. al. Rural-urban inequalities in detection rates of colorectal tumours in the population. Dig. Liver Dis. 2012; 44: 172-177.

18. Schootman M, Lian M, Deshrande A, McQueen A, PRUITT S, JefFE D. Temporal trends in geographic disparities in small-area-level colorectal cancer incidence and mortality in the United States. Cancer Causes Control 2011; 22: 1-9.

19. Giovannucci E, Wu K. Cancers of the colon and rectum. In Schottenfeld D, Fraumeni JF (eds): Cancer Epidemiology and Prevention. New York, NY: Oxford University Press 2006; 809829 . 
20. BéJar L, Gili M, Díaz V, Ramírez G, López J, CABANILLAS JL et al. Incidence and mortality by colorrectal cancer in Spain during 1951-2006 and its relationship with behavioural factors. Eur J Cancer Prev 2009; 18: 436-444.

21. López-Abente G, Ardanaz E, Torrella-Ramos A, Mateos A, Delgado-Sanz C, Chirlaque MD et al. Changes in colorrectal cancer incidence and mortality trends in Spain. Ann Oncol 2010; 21: 76-82.

22. National Cancer Institute. Colorectal Cancer Prevention. http://www.cancer.gov/cancertopics/pdq/prevention/colorectal/HealthProfessional (fecha consulta 12/04/2012).

23. Karim-Kos HE, De Vries E, Soerjomataram I, LemMENS V, Siesling S, CoEbergh JWW. Recent trends of cancer in Europe: a combined approach of incidence, survival and mortality for 17 cancer sites since the 1990s. Eur J Cancer 2008; 44: 1345-1389.

24. Garcia-Closas R, Berenguer A, GonzÁlez CA. Changes in food supply in Mediterranean countries from 1961 to 2001. Public Health Nutr 2006; 9: 53-60.

25. Viñes JJ, Ardanaz E, Arrazola A, Gaminde I. Population-based epidemiology of colorrectal cancer: causality review. An Sist Sanit Navar 2003; 26: 79-97.

26. Sant M, Allemani C, Santaquilani M, Knijn A, Marchesi F, Capocaccia R et al. EUROCARE-4. Survival of cancer patients diagnosed in 1995-1999. Results and commentary. Eur J Cancer 2009; 45: 931-991.
27. Ribes J, Navarro M, Clèries R, Esteban L, Pareja L, BINEFA G et al. Colorrectal cancer mortality in Spain: trends and projections for 19852019. Eur J Gastroenterol Hepatol 2009; 21: 92-100.

28. Ciccolallo L, Hakulinen T, Lemmens VE and EUROCARE - Working Group. Evaluation of standard of care for colorectal cancer patients using EUROCARE high resolution data 2007. En: Actas de la XXXII Reunión del Grupo para la Epidemiología y el Registro del Cáncer en los Países de Lengua Latina, (Montreal (Canadá) del 16 al 19 de mayo de 2007), p. 75.

29. Ugarte MD, Goicoa T, Etxeberria J, Militino AF, Pollán M, Age-specific spatio-temporal patterns of female breast cancer mortality in Spain (1975-2005). Ann Epidemiol 2010; 20: 906-916.

30. El Consejo de la Unión Europea. Recomendación del Consejo de 2 de Diciembre de 2003 sobre el cribado del cáncer. Diario Oficial de la Unión Europea 2003; 327: 34-37.

31. Borrás JM, Colomer C, Soria P, López R. Priorities for cancer control in Spain Ann Oncol 2010; 21: 111-114.

32. López-Abente G, Pollán M, Vergara A, Ardanaz E, Moreo P, Moreno C et al. Tendencia temporal de la incidencia de cáncer en Navarra y Zaragoza. Gac Sanit 2000; 14: 100-109.

33. Navarro C, Martos C, Ardanaz E, Galceran J, Izarzugaza I, Peris-Bonet R et al. Population-based cancer registries in Spain and their role in cancer control. Ann Onc 2010 21: 3-13. 
